\title{
Applying health promotion theories to improve depressive symptoms through exercise
}

\author{
Dor David Abelman, Andrew Daniel Hanna \\ Faculty Reviewer: Jennifer D Irwin, PhD, MA, BA (School of Health Studies)
}

\begin{abstract}
Considering both the high monetary and emotional costs associated with caring for patients afflicted with depression, it is apparent that health professionals should find effective treatments to ensure that interventions are both cost effective and risk-reducing. While exercise is generally known to be important in maintaining general health and wellbeing, numerous studies have also indicated that it can play a critical role in moderating symptoms of mild to moderate depression. Globally, research continues to show a powerful reduction in depressive symptoms due to the benefits of exercise. This review article will highlight the growing perspective regarding the influence of exercise as a first-line treatment for depression over pharmacotherapy for patients with mild to moderate depression. Health promotion theories including the Theory of Planned Behaviour, Theory of Reasoned Action, and Motivational Interviewing will be applied.
\end{abstract}

\section{BACKGROUND}

In 2007, an article in Psychosomatic Medicine highlighted the impact of physical activity on depressive symptoms, suggesting that its effects may be comparable to antidepressants in a five-year prospective cohort study of 200 adults. ${ }^{1}$ Over the next few years, both a systematic review and Cochrane review suggested that exercise was as effective as pharmacotherapy, if not more effective, as a treatment method for mild to moderate depression. ${ }^{2,3}$ Various additional studies have found that a correlation exists between physical inactivity and depressive symptoms. ${ }^{4,5}$ Worldwide, approximately 340 million people are currently afflicted with depression. ${ }^{2}$ Given that depression is projected to be the second leading burden of disease by 2020 , it stands to reason that a variety of approaches are required to best address this issue for patients and the healthcare system alike. ${ }^{2}$

The benefits of exercise are diverse and numerous. The Public Health Agency of Canada (PHAC) recommends that Canadian adults be active for at least 2.5 hours every week, combining both aerobic and resistance training. ${ }^{6}$ PHAC also advocates that exercise provides secondary health benefits to patients with depression such as, but not limited to, substantially reducing the risk of chronic diseases and various cancers - and by extension, mortality. ${ }^{6,7}$ These findings are reflected in the literature and are applicable at all ages. ${ }^{8,10}$

\section{CONTEMPORARY ISSUES}

The Canadian Health Measures Survey suggests that less than one quarter of Canadians are sufficiently active. ${ }^{11}$ More troubling is the finding that only $9 \%$ of children and youth get their minimum physical activity recommendations for optimal growth and wellbeing. ${ }^{12}$ Patients have often reported lack of motivation, commonly induced by their depressive symptoms, as one of the major barriers to actually engaging in physical activity. ${ }^{13,14}$ As such, this lack of patient motivation actually creates yet another barrier as physicians become reluctant to prescribe exercise, citing preconceived concerns about patient disinterest in engaging in physical activity on a regular basis. ${ }^{13}$

A qualitative study on patient perceptions regarding the potential reduction of depressive symptoms due to physical activity suggested that patients who perceived exercise to be a positive force in reducing depressive symptoms actually enjoyed significant benefits. ${ }^{14}$ Furthermore, the study reported that exercise also helped to improve patients' eating habits, social interactions, and sleep patterns - factors which indirectly improved their mood, and ultimately, reduced their depressive symptoms. ${ }^{14}$

Taking all these factors into account, it is important to improve both the public's perception of exercise and their motivation to engage in physical activity. This shift would theoretically help to relieve the reluctance of physicians in prescribing exercise, thereby allowing them to more successfully encourage patients to exercise in a way that improves depressive symptoms.

\section{PHYSICIANS PLAY AN IMPORTANT ROLE IN ENCOURAGING EXERCISE BEHAVIOUR BUT COULD BE MORE SUCCESSFUL IN THIS TASK IF HEALTH PROMOTION PRINCIPLES WERE IMPLEMENTED.}

\section{LEARNING FROM HEALTH PROMOTION}

The difficulties associated with encouraging the public to exercise are well-known and well-researched in the field of health promotion. ${ }^{15,16}$ This section will discuss and examine several theories and models that may work to encourage the public to engage in exercise as a means of reduce and prevent depressive symptoms.

The Theory of Reasoned Action (TRA), developed by Ajzen \& Fishbein, helps one predict how an individual will behave by describing the components that lead to their behavioural decisions. ${ }^{17}$ Reasoned action refers to the thought process one goes through to choose their behaviours. ${ }^{17}$ The theory's two main components are personal beliefs and subjective norms. ${ }^{17}$ Subjective norms can be 
further broken down to normative expectations (what others expect of an individual) and motivation to comply (the importance of doing what others expect)..$^{1718}$ The theory was innovative for its emphasis on the importance of subjective norms such as social acceptance and perceived social support to complete a behaviour. ${ }^{17}$

When applied to exercise, TRA suggests that peer and community supports (subjective norms) are essential contributors to an individual's desire to exercise. ${ }^{18}$ This directly relates to the challenge reported in a review done by Blake et al - that physicians were reluctant to prescribe exercise because their patients had preconceived notions about the importance of adhering to an exercise routine. ${ }^{13}$ In other words, the patients may not have the social support that the TRA says is essential to motivate them to exercise, as per physician recommendation. ${ }^{17,18}$

To address the lack of social support to exercise or abide by physician requests, health education campaigns that portray exercise or abiding by physician requests in a positive manner should be implemented. Changing the general public's perception on this topic may indirectly promote social support to comply to the behaviour. ${ }^{19}$ These campaigns should focus on the importance of exercise and trusting physicians. Furthermore, they could be more effective if they promote healthy subjective norms of normative expectations and motivation to comply. ${ }^{17}$ Physicians can consider the principles of TRA while helping a patient understand that their actions of exercise are socially acceptable (normative expectations) or very important (motivation to comply). ${ }^{20}$ This can be done through displaying media that promotes physical activity to be more appealing, or counselling a patient about its significance for health and wellbeing. ${ }^{20}$

Another relevant theory for health promotion is the Theory of Planned Behaviour (TPB), an adapted version of the TRA which adds an additional component into the process of modifying behaviour - a person's perceived control of the behaviour change. ${ }^{18}$ Planned behaviour describes the process one uses to plan and fulfill the actions they take. ${ }^{20}$ The theory identifies perceived opportunities, skills, and resources as important components of assessing control. ${ }^{18}$ The difference between an individual's perceived opportunities, skills, and resources and the amount they think is required to make a behaviour change is important to predict their chance of complying to a behaviour change request. ${ }^{18}$ This theory, then, purports the notion that in commencing an exercise regimen, it is paramount that patients feel they are able to participate in activities which would provide them with a sufficient amount of agency and enjoyment. ${ }^{20}$ Such a notion could come to fruition through a physician acknowledging the personal interests and preferences of patients, and by implementing these into their exercise plan so as to make it more palatable and enjoyable for patients. ${ }^{20}$

Lastly, another key approach to health promotion is the Motivational Interview, which is defined as a "client-centered counseling style that helps people to explore and resolve their ambivalence regarding [behaviour] change." ${ }^{21}$ The key components of motivational interviewing are open-ended questions, affirmation (sincere acknowledgement of a patient's concerns to validate their experiences), reflective listening (demonstrating understanding of a patient's communication by restating its meaning), and periodical summarization (distilling and reiterating select communications from a patient to acknowledge their ideas and to help guide them to move on). ${ }^{22}$ Motivational interviewing is different from traditional patient interviews because of its focus on a patient's ability to make their own choices through self-actualization. ${ }^{22}$ This is achieved through helping a patient identify discrepancies between their current position and where they hope to be, while promoting their perceived self-efficacy to achieve this goal. ${ }^{22}$

Those attempting to incite any kind of behavioural change namely, patient attitudes towards abiding by a prescribed exercise regimen - will be privy to individual needs through counselling sessions, during which counselors should: show empathy, listen respectfully, avoid conflict, and promote means of resolving a patient's ambivalence towards engaging in physical activity. ${ }^{22} \mathrm{~A}$ meta-analysis has already demonstrated motivational interviewing to be successful for inciting behavioural changes in a variety of contexts, and it is not so far-fetched to claim that it could also be effective in this particular context. ${ }^{23}$ A 2014 randomized control trial found that primary care physicians trained with principles of motivational interviewing were better able to encourage their patients with depression to be interested in treatment options and adhere to them. ${ }^{24}$

\section{CONCLUSION}

Physicians play an important role in encouraging exercise behaviour but could be more successful in this task if health promotion principles were implemented. The Theory of Reasoned Action, the Theory of Planned Behaviour, and Motivational Interviewing are evidence-based strategies that have been applied successfully in a variety of contexts to incite behaviour change. More research in this area could be applied to find the best way to increase exercise as therapy for depression and reduce what could soon be the world's second largest burden of disease.

\section{ACKNOWLEDGEMENTS}

We would like to thank Dr Jennifer Irwin and Rebecca Meharchand for their support, time, and help in completing this project.

\section{REFERENCES}

1. Blumenthal JA, Babyak MA, Doraiswamy PM, et al. Exercise and pharmacotherapy in the treatment of major depressive disorder. Psychosom Med. 2007;69(7):587-96.

2. Josefsson T, Lindwall M, Archer T. Physical exercise intervention in depressive disorders: Meta-analysis and systematic review. Scand J Med Sci Sports. 2014 Apr;24(2):259-72.

3. Cooney GM, Dwan K, Greig CA, et al. Exercise for depression (review) summary of findings for the main comparison. Cochrane Database Syst Rev. 2013;(9).

4. Galper DI, Trivedi MH, Barlow CE, et al. Inverse association between physical inactivity and mental health in men and women. Med Sci Sports Exerc. 2006 Jan;38(1):173-8. 
5. Craft LL, Perna FM. The Benefits of Exercise for the Clinically Depressed. Prim Care Companion J Clin Psychiatry. 2004;6(3):104-11.

6. Public Health Agency of Canada. Physical Activity Tips for Adults (18-64 years) - Tips to Get Active - Physical Activity - Public Health Agency of Canada [Internet]. Physical Activity. 2012 [cited 2017 Mar 23]. Available from: http://www.phac-aspc.gc.ca/hp-ps/hl-mvs/paap/07paap-eng.php.

7. Statistics Canada. The 10 leading causes of death, 2011 [Internet]. Health Fact Sheets. 2015 [cited 2017 Apr 4]. Available from: http:// www.statcan.gc.ca/pub/82-625-x/2014001/article/11896-eng.htm.

8. Gregory MA, Gill DP, Shellington EM, et al. Group-based exercise and cognitive-physical training in older adults with self-reported cognitive complaints: The Multiple-Modality, Mind-Motor (M4) study protocol. BMC Geriatr. 2016 Dec 16;16(1):17.

9. Bauman A, Merom D, Bull FC, et al. Updating the Evidence for Physical Activity: Summative Reviews of the Epidemiological Evidence, Prevalence, and Interventions to Promote "active Aging." Vol. 56, Gerontologist. 2016. p. S268-80.

10. Anand P, Kunnumakkara AB, Sundaram C, et al. Cancer is a preventable disease that requires major lifestyle changes. Pharm Res. 2008 Sep;25(9):2097-116.

11. Statistics Canada. Directly measured physical activity of adults, 2012 and 2013 [Internet]. Canadian Health Measures Survey. 2015 [cited 2017 Apr 4]. Available from: http://www.statcan.gc.ca/pub/82625-x/2015001/article/14135-eng.htm.

12. ParticipACTION. Are Canadian kids too tired to move? The 2016 ParticipACTION Report Card on Physical Activity for Children and Youth [Internet]. 2016 [cited 2017 Apr 4]. Available from: https://www. participaction.com/sites/default/files/downloads/2016 ParticipACTION Report Card - Highlight Report.pdf.

13. Blake H. Physical activity and exercise in the treatment of depression. Front Psychiatry. 2012;3(DEC):106.

14. Searle A, Calnan M, Lewis G, et al. Patients' views of physical activity as treatment for depression: a qualitative study. Br J Gen Pract. 2011 Apr;61(585):149-56.

15. Teixeira PJ, Carraça E V, Markland D, et al. Exercise, physical activity, and self-determination theory: a systematic review. Int J Behav Nutr Phys Act. 2012 Jun 22;9:78.

16. Khatta M. Models and Interventions to Increase Physical Activity Among Adults: A Historical Review. Top Adv Pract Nurs . 2008;8(1)

17. Ajzen I, Fishbein M. Understanding attitudes and predicting social behavior. NJ: Prentice Hall: Englewood Cliffs; 1980. 278 p.

18. US Department of Health and Human Services. Appendix 3: Theories and Models Used in Physical Activity Promotion. Physical activity evaluation handbook. Atlanta, GA; 2002.

19. Centers for Disease Control and Prevention. Appendix 2: Guide to Community Preventive Services Recommendations. Physical activity evaluation handbook. Atlanta. GA; 2002.

20. Godin G. The theories of reasoned action and planned behavior: Overview of findings, emerging research problems and usefulness for exercise promotion. J Appl Sport Psychol. 1993;5(2):141-57.

21. Miller WR, Rollnick S. Motivational Interviewing: preparing people for change. 2nd ed. New York: The Guilford Press; 2002. 428 p.

22. Center for Substance Abuse Treatment. Enhancing Motivation for Change in Substance Abuse Treatment. Rockville (MD): Substance Abuse and Mental Health Services Administration (US); 1999. (Treatment Improvement Protocol (TIP) Series, No. 35.) Chapter 3- Motivational Interviewing as a Counseling Style.

23. Rubak S, Sandbaek A, Lauritzen T, et al. Motivational interview- ing: a systematic review and meta-analysis. Br J Gen Pract. 2005 Apr;55(513):305-12.

24. Keeley RD, Burke BL, Brody D, et al. Training to Use Motivational Interviewing Techniques for Depression: A Cluster Randomized Trial. J Am Board Fam Med. 2014 Sep 1;27(5):621-36. 